\title{
Augmented bio-based lipids for cosmetics
}

\author{
Sébastien Duprat-de-Paule*, Jérôme Guilbot, Alicia Roso, Sophie Cambos and Aurélie Pierre \\ SEPPIC, 22 Terrasse Bellini, Paris La Défense, 92800 Puteaux, France
}

Received 24 April 2018 - Accepted 15 June 2018

\begin{abstract}
Practical examples showcase the key role of plant-based lipids in the design of innovative sustainable specialty ingredients. Great diversity in plant origins and chemical transformations leads to great molecular diversity and explains why bio-based lipids are involved in broad ingredient categories such as biodegradable emollients, environmentally friendly surfactants, rheology modifiers and active ingredients. Choosing lipid structure, with varying fatty chain length, saturation level and branching, determines ingredient functionality and usage, as these vary, for instance in the case of surfactants, solubilizing, wetting, foaming and emulsifying properties (oil-in-water or water-in-oil). The lipid structure also impacts the ingredients' final solid or liquid appearance. Now ready-to-use ingredients can be created and we can innovate with cold processable new cosmetic formulation concepts. Perhaps most importantly, optimal selection of lipid structure and composition can also drive consumer benefits in cosmetic ingredients, especially, the final sensory experience (for excipients) and biological efficacy (for active ingredients). Biobased lipids lead to new ingredients with augmented performance and sensoriality.
\end{abstract}

Keywords: ingredient / emollient / surfactant / efficacy / sensoriality

\begin{abstract}
Résumé - Des lipides biosourcés à forte valeur ajoutée pour la cosmétique. Cet article vise à illustrer à travers des exemples concrets le rôle clé des lipides biosourcés dans la création d'ingrédients de spécialités innovants et durables. La grande variété des plantes d'origine et la diversité des procédés de transformation chimiques expliquent la large implication des lipides biosourcés dans de nombreuses catégories d'ingrédients: les émollients et tensio-actifs respectueux de l'environnement, les modificateurs de rhéologie, ainsi que les ingrédients actifs. Le choix de la structure lipidique tel que la longueur de la chaîne grasse, son taux de saturation ou son caractère branché joue un rôle décisif dans la fonctionnalité de l'ingrédient et son usage comme, par exemple, en tant que solubilisant, mouillant, moussant ou émulsionnant (huile-dans-eau ou eau-dans-huile) pour les tensioactifs. La structure lipidique influence aussi l'aspect liquide à solide de l'ingrédient et permet ainsi de créer des ingrédients prêts à l'emploi et d'innover avec des concepts de formulation cosmétique à froid. Enfin, et cela est probablement le plus important, une choix judicieux de la structure et de la composition lipidique peut aussi orienter les bénéfices de l'ingrédient pour le consommateur final, en particulier l'expérience sensorielle pour les excipients et l'efficacité biologique pour les ingrédients actifs. Les lipides biosourcés sont à l'origine de nouveaux ingrédients avec toujours plus de performance et de sensorialité.
\end{abstract}

Mots clés : ingrédient / emollient / tensioactif / efficacité / sensorialité

\section{Introduction}

Oleochemistry is at the root of oil-based ingredients in many fields: in industry as biosolvents, biofuels or biolubricants, in the food sector as excipients or nutritional supplements, in pharmaceuticals, to name just a few. Plant-based lipids are also essential and invaluable raw materials in the design of sustainable specialty ingredients for health and beauty (e.g. triglycerides, fatty acids and alcohols, and plant waxes).

\footnotetext{
*Correspondence: sebastien.dupratdepaule@airliquide.com
}

Lipids are very diverse and have been classified in different ways. They are also obtained in diverse ways: from simple plant mechanical expression processes to more complex chemical transformations.

Figure 1 gives a simplified overview of the lipid processing chain from the perspective of an ingredient manufacturer. Depending on their nature, lipids along the processing chain can be used either directly as ingredients in final cosmetic compositions or as raw materials for the production of advanced cosmetic ingredients. Plant oils and butters have been, for instance, recognized for centuries as efficient 


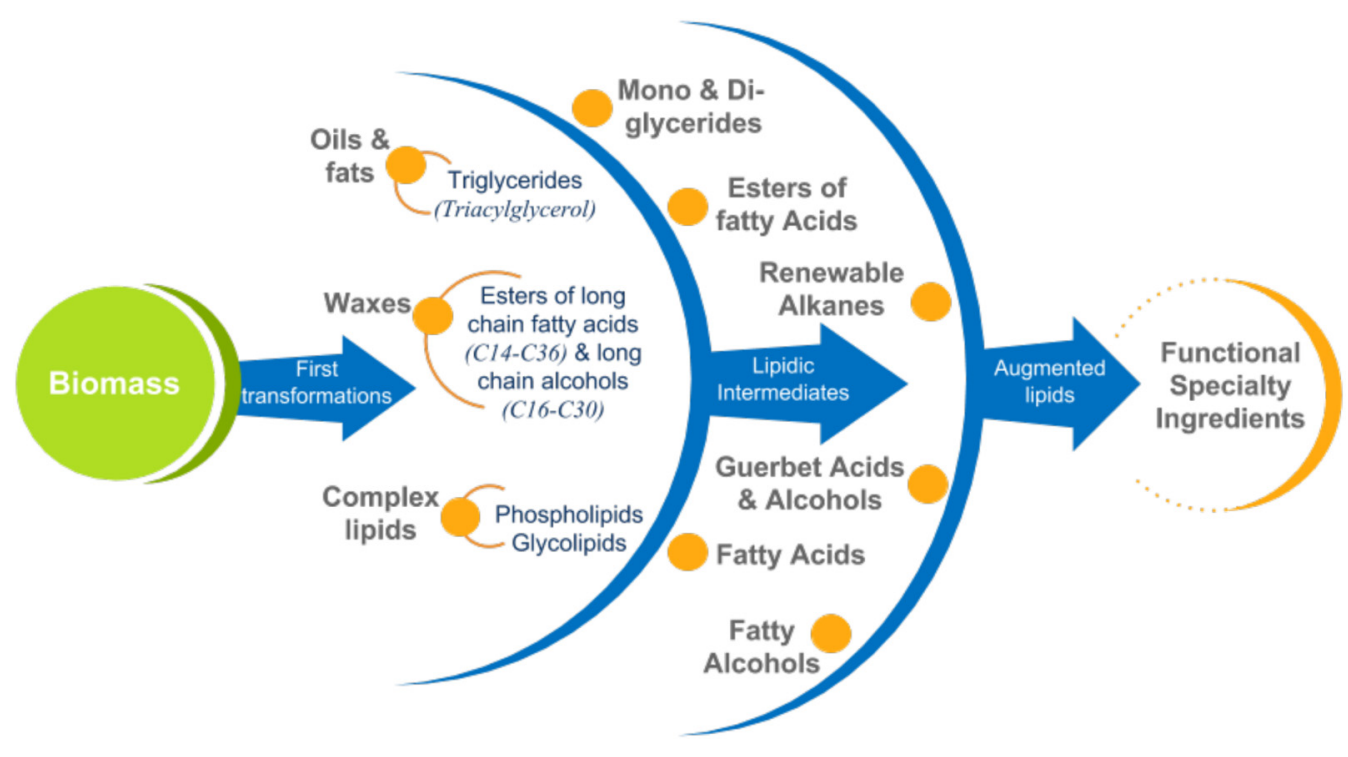

Fig. 1. Lipid processing chain summary.

emollients. Plant waxes, fatty acids and alcohols are widely used as texturizing agents in emulsions, to provide consistency. Since the 1970s, fatty acids and alcohols have also been used as raw materials in surfactant design.

Beyond traditional uses, cosmetic and dermocosmetic manufacturers are increasingly looking for specialty ingredients with added value, such as easier, quicker handling procedures. At the same time, the last decade has seen growing consumer environmental concerns as well as increasing expectations concerning both formulation efficacy and aesthetics. More than only a product, consumers are nowadays indeed looking for a global experience, in total accordance with their lifestyle.

From this perspective, here we highlight the importance of bio-based-lipid derivatives in driving ingredient functionality and performance among broad categories: emollients, rheology modifiers, surfactants and active ingredients. Three areas are considered for these multifaceted lipids' key contribution:

- environmental footprint;

- performance targets;

- distinctive sensory profiles.

\section{Lipids, inspiring drivers for sustainable innovation}

The plant world offers chemists and formulators lipids characterized by a large variety of hydrocarbon chains from oleochemistry. First oilseed crops are grown (See Fig. 2, palm, coconut, olive, castor, rape, etc.) and, after harvest, then the triglycerides they contain are extracted. These triglycerides correspond to three hydrocarbon chains linked to a glycerol moiety by three ester bonds. Depending on the nature of the oilseed crops, these hydrocarbon chains are very different, though they always have an even number of carbons between 8 and 22. They may have naturally occurring unsaturations $(1,2$ or 3 ) or branching after first chemical transformations. A plant never contains a pure triglyceride with a single type of chain but rather a complex mixture whose average weight ratios can be estimated according to the initial plant as described in Figure 2. Plant triglycerides are rarely chemically functionalized. The only example of that is castor oil, which is characterized by $\mathrm{C}-18$ chains with a $9-10$ double bond and also a hydroxyl function at the $\mathrm{C}-12$ position.

In a second step, these triglycerides can be hydrolyzed either in presence of a base (saponification) or in the presence of methanol and a base (methanolysis). These processes produce respectively soaps (or carboxylic acids after neutralization) and methyl esters, which are sources of lipids for formulators and chemists.

In a third step, these carboxylic acids and methyl esters can finally be modified, leading to various lipophilic chains such as fatty alcohols (hydrogenation), fatty amines $\left(\mathrm{NH}_{3}\right.$ treatment) or fatty acyl chlorides (chlorinating agent). Another way to widen the lipid offering is to cleave the unsaturated triglycerides' double bonds. For instance, oleic acid ozonolysis forms azelaic acid (a dicarboxylic acid) and pelargonic acid (a natural weed killer). Another example is the thermal treatment of ricinoleic acid under basic conditions leading to sebacic acid (a dicarboxylic acid) and 2-octanol (at $250^{\circ} \mathrm{C}$ ) or undecenyl acid and heptaldehyde (at $400^{\circ} \mathrm{C}$ ).

In nature, other lipids than triglycerides are also extracted from vegetables. Their chemical structure corresponds to lipophilic esters, also called wax esters, available as liquids or solids. The most famous liquid wax is jojoba oil characterized by C36 to C46 chains. Finally we can also quote other examples of solid waxes such as candelilla and carnauba waxes that are generally directly used to bring consistency and for their emollient performance.

\subsection{New emollients, biodegradable alternatives to silicone oils}

Research programs on sustainable palm oil biomass allowed to develop fully bio-based non-polar alkanes as a "sensory alternative" to silicone oils. Initially dedicated to skincare applications, these emollients (e.g. the Emogreen ${ }^{\mathrm{TM}}$ range) have also revealed promising potential in haircare 
- Palm Kernel Oil C8 - C18 \& Coconut Oil
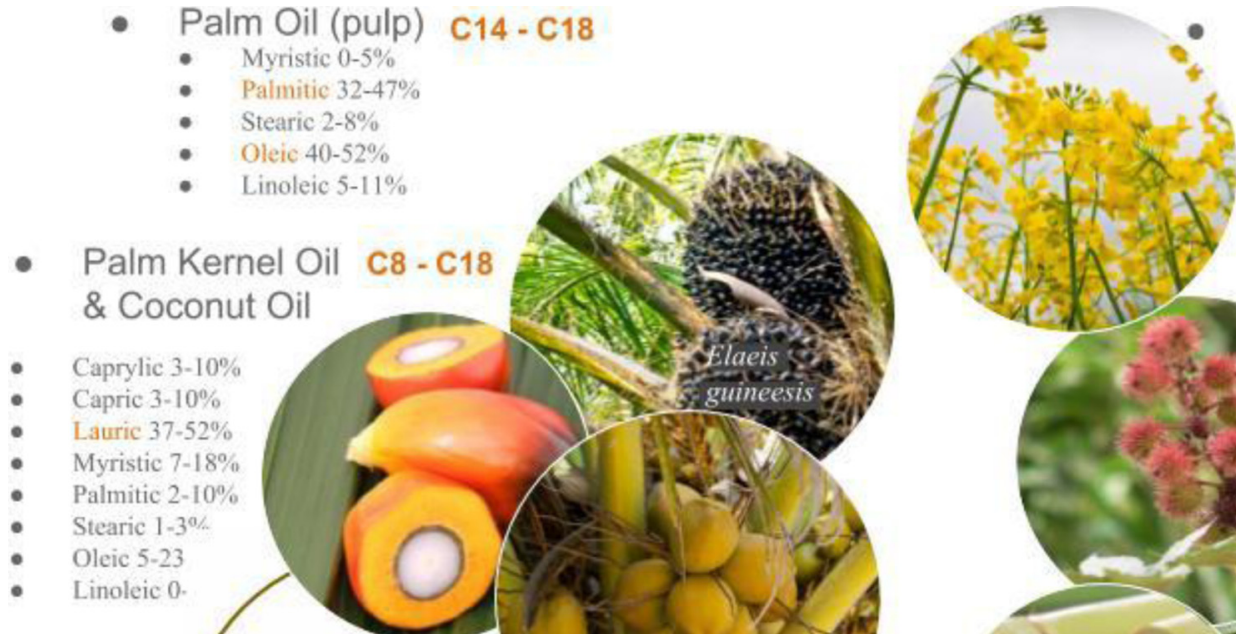

Rapeseed C16-C22

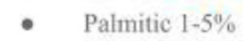

- Stearic 0-2\%

- Olcic 50-65\%

- Linoleic 15-30\%

- Linolenic 6-13\%

- Eicosenic 1-3\%

- Behenic 0-1\%

$\bullet$
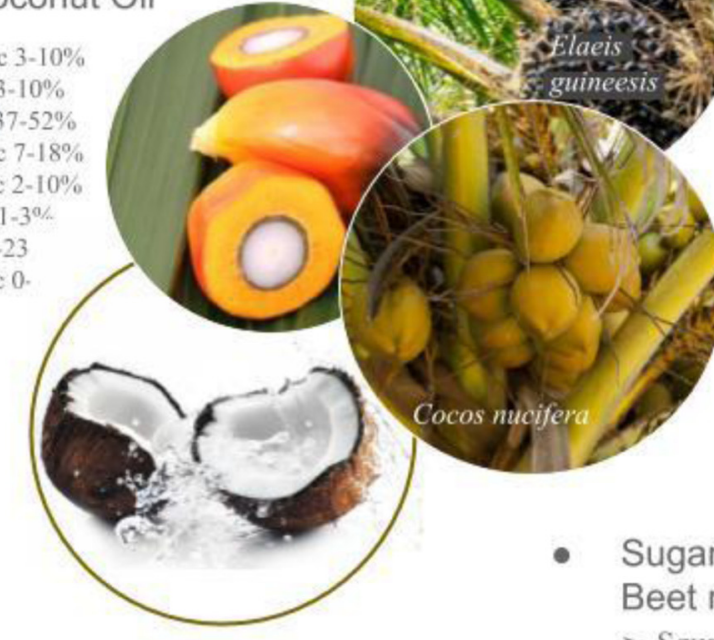


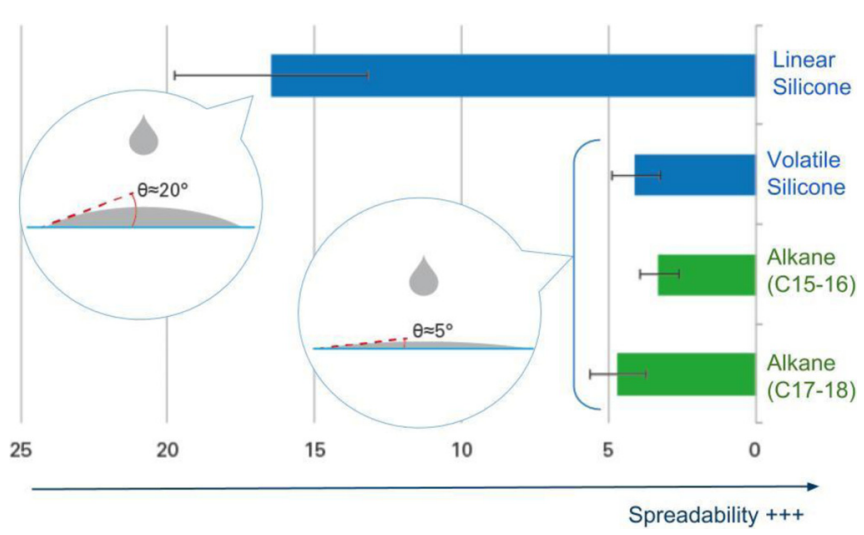

Fig. 3. Oil contact angle at 0.8 seconds.

and allow formulation in extreme conditions (e.g. a wide $\mathrm{pH}$ range).

Performances of alkanes as "sensory alternatives" to silicones in skin care has been challenged on the pure oils as well as in formulations. Good spreading is expected from the low surface tension but as spreading of cosmetics can be decomposed into different steps the two parameters have to be evaluated in more realistic conditions. The steps are firstly, how the formulation wets the skin and secondly, how well the formula glides on the skin under hand pressure (Nonomura et al., 2009). To confirm that pure alkane oils have good wetting properties with fast spontaneous spreading, similar to the tested volatile silicone, low contact angle values are measured on a polymethyl methacrylate rough surface plate, used to mimic the skin (PMMA Sunplates, Helioscience, Digidrop Fast $/ 30$ GBX goniometer, average of 3 measures) (Savary et al., 2013). Figure 3 shows comparative values at 0.8 seconds, chosen to avoid any impact from oil volatility. Easy gliding of the alkanes when submitted to a rotating movement under pressure is also demonstrated by low friction coefficients, in between the two silicone oils. Figure 4 shows the friction coefficients after application of a $90 \mu \mathrm{m}$ layer of each oil on an aluminium plate, measured with a new tribology accessory, called ring on plate geometry, fixed on a hybrid rheometer (DHR2, TA Instrument) (Latshaw, 2014). Assessments were performed with an increasing velocity gradient between 0.01 and $15 \mathrm{radian} / \mathrm{second}$ with a fixed $2 \mathrm{~N}$ load force. The $10 \mathrm{rad} / \mathrm{s}$ velocity was selected for comparative illustration as the closest condition to a real spreading protocol on skin. In a final step, the oils were added at $15 \%$ to a standard cosmetic gel formula or at $9 \%$ to a standard oil-in-water $(\mathrm{O} / \mathrm{W})$ emulsion, and in vivo sensory evaluation was done with 10 to 13 trained expert panelists. An emulsion consists of very fine droplets of a liquid dispersed in a second immiscible liquid: oil droplets dispersed in water for oil-in-water emulsions or water droplets dispersed in oil for water-in-oil (W/O) emulsions. The results, consistent with the chemical measurements, show similar spreadability of alkanes as for the tested silicones (See one illustrative compared sensory profile in Fig. 5) whereas the C17-18 alkane is judged, after statistical analysis, as being less dry with a more comfortable afterfeel than the volatile silicone oil. Within the same cream gel and emulsion formulas, its sensoriality is ultimately recognized by hundreds of female consumers as the most preferred among six different oils,

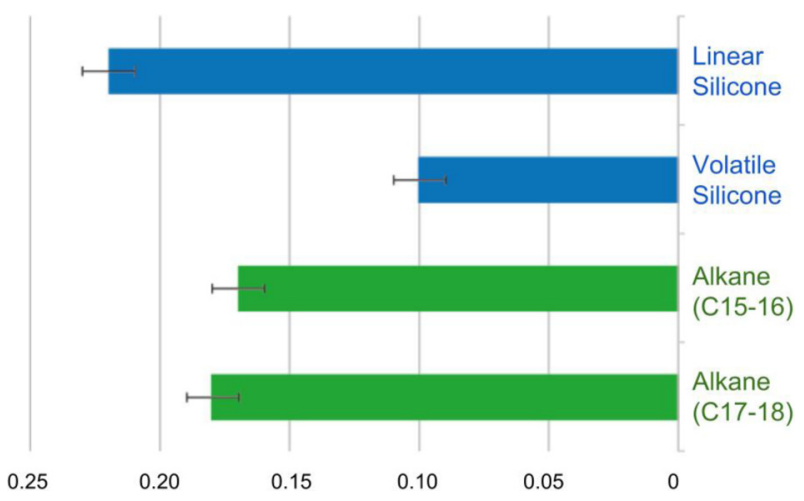

Fig. 4. Oil friction coefficient at $10 \mathrm{radian} / \mathrm{second}$.

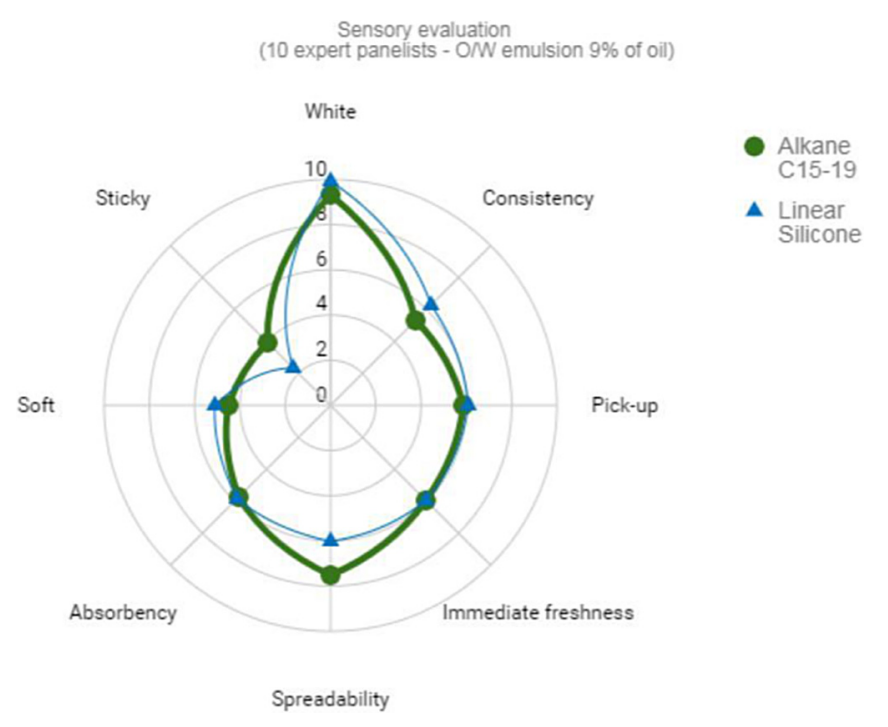

Fig. 5. Example of sensory profile.

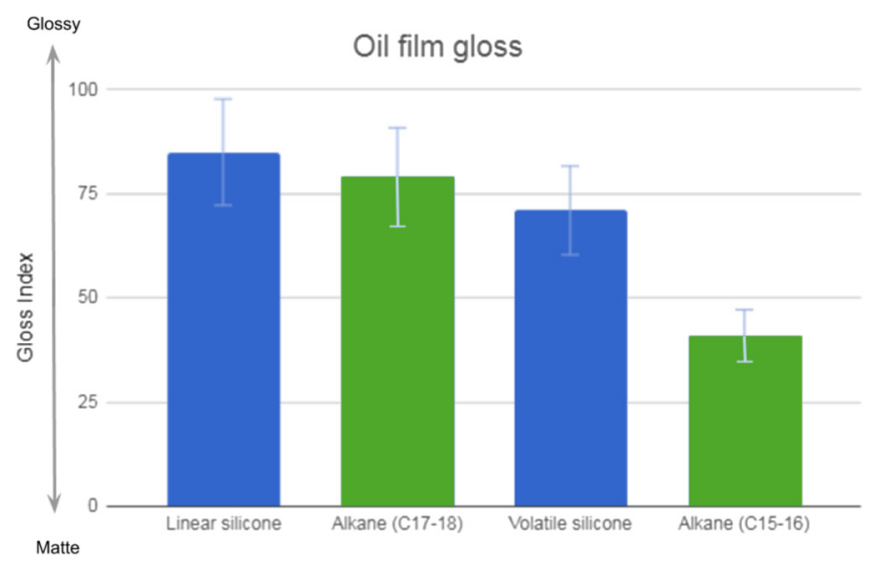

Fig. 6. Alkane film gloss.

including the linear silicone and bio-based esters such as jojoba and $\mathrm{C} 8$ to $\mathrm{C} 10$ triglycerides. Keeping in mind other well known properties of silicone oils, matte finish was also compared one minute after spreading $0.5 \mathrm{ml}$ of pure oils on a polymethyl methacrylate plate (PMMA Sunplates, Helioscience). Film gloss level was measured with a gloss meter 


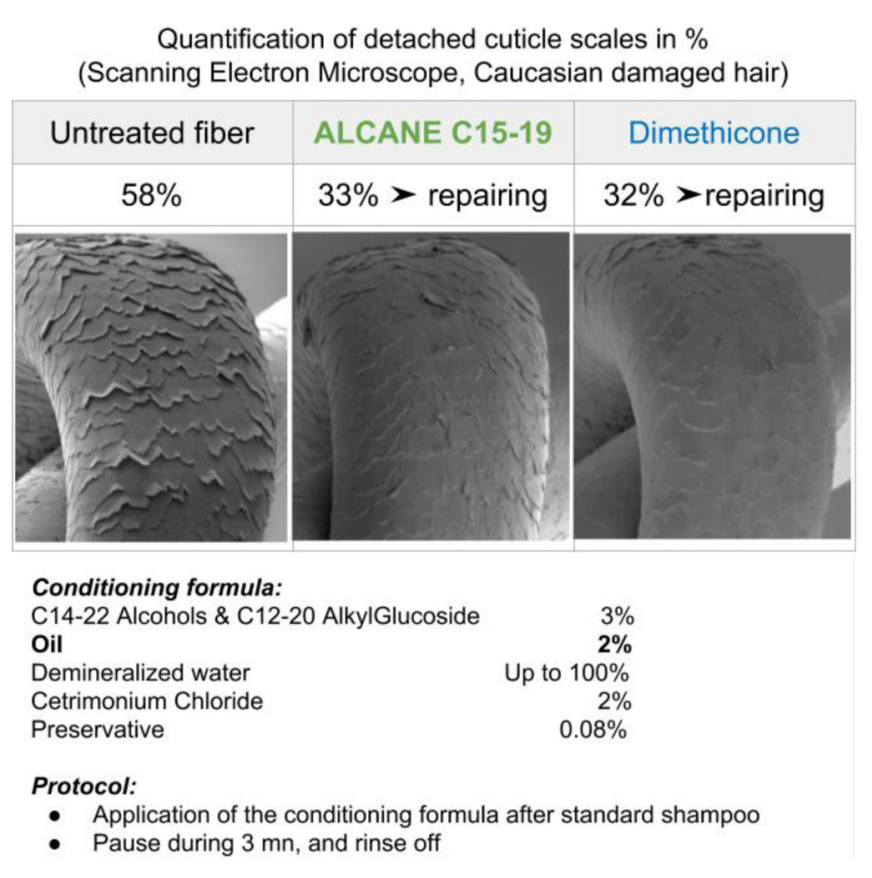

Fig. 7. Alkane repairing effect on damaged hair fiber.

(Micro Tri-Gloss 4520, Byk Gardner; angle: $60^{\circ}$, gloss index from 0 to 100 for highest gloss level, average of 5 measures). The results shown in Figure 6 highlighted matte appearance provided especially by the shortest C15-16 alkane, in line with a gloss index even lower than the volatile silicone reference. Moreover, C15-16 alkane helps to reduce sticky sensation perceived with $\mathrm{O} / \mathrm{W}$ emulsions containing high concentration of glycerine ( $10 \%$ tested), suncare formulas with high level of sunscreens as well as after lip oil application. Deeper investigations of alkanes' behavior in suncare and make-up should be conducted to further determine if alkanes enable to similar long lasting performance than silicone oils.

Looking to environmental context, even more critical for wash-off cosmetic products such as hair conditioners, these bio-based alkanes are also promising alternatives. A first exploratory test compared to the linear silicone, at $2 \%$ in a $\mathrm{O} / \mathrm{W}$ hair conditioning emulsion applied on bleach hair tresses, indeed shows similar efficacy on wet combing, volume and frizz control as well repairing damaged surface fibres (Fig. 7).

These new environmental-friendly emollients open new possibilities for the responsible cosmetic market, allowing diversification of oil combinations and optimization of textures and skin feel.

\section{Lipids offer access to ready-to-use ingredients, for more sustainable formula manufacturing processes}

The choice of lipid structure, such as saturation level and branched character, is very important in designing innovative ready-to-use ingredients. Therefore, a well-chosen lipid raw material can be the way into new formulation concepts, achieved by a cold process, that enable both time savings and an improved manufacturing environmental footprint.
In cosmetic products, $\mathrm{O} / \mathrm{W}$ or $\mathrm{W} / \mathrm{O}$ emulsions are the most used formulation type. They are indeed good vehicles for active ingredients and they provide various sensory profiles that are easily customizable to the final application target. However, they have weaknesses linked to hot manufacturing procedures and/or high shear mixing requirements, sometimes combined, especially for $\mathrm{W} / \mathrm{O}$ with the need of progressive oil addition, which slows down the manufacturing. Suitable liquid, ready-to-use ingredients can overcome these issues without compromising cosmetic sensoriality.

Lipid selection plays a key role in the design of suitable liquid ingredients, especially to create efficient liquid emulsifiers and liquid rheology modifiers.

\subsection{Liquid W/O emulsifier}

The first example involves glycolipid surfactants, obtained by a glycosylation reaction between a fatty alcohol and a sugar. These represent a major bio-based surfactant category. Long linear chain fatty alcohols condensed with glucose produce efficient $\mathrm{O} / \mathrm{W}$ emulsifiers presented in solid pearl form. To design a liquid emulsifier starting with the same idea, a branched guerbet alcohol (octyldodecanol) was selected and glucose was replaced by xylose to increase chemical reactivity (Fig. 8). As mentioned before, branched alcohols are not naturally available. Nevertheless, octyldodecanol biosourcing can be claimed because it comes from the thermal dimerization of two natural linear C-10 alcohols in the presence of clay as catalyst. Since branched alcohols have much lower melting points than linear alcohols at equivalent fatty chain length, the final ingredient, a mixture of octyldocecyl polyxyloside and octyldodecanol is liquid at room temperature (e.g. the Fluidanov $^{\mathrm{TM}}$ range). This ingredient is an easy-to-use $\mathrm{W} / \mathrm{O}$ co-emulsifier that benefits from small molecular size to quickly migrate to the water/oil interface while providing a soft and silky skin feel. To optimize W/O emulsion stability, this biobased liquid co-emulsifier was finally combined with a high molecular weight polymeric surfactant, PEG-30 dipolyhydroxystearate, to provide a ready-to-use W/O emulsifying base and keep the liquid form. Other polymeric surfactants such as Polyglyceryl-2 Dipolyhydroxystearate can also be used but its versatility to stabilize the emulsion is more sensitive to the oil nature.

\subsection{Liquid rheology modifier}

As a second example, suitable oil selection is at the root of radical inverse polymerization technology. The technology consists in first making a stable W/O emulsion with hydrophilic monomers added to the aqueous phase. The polymerization process is then handled directly inside the water droplets after the initiator or redox agents are added. The rheology modifier is post-added with high HLB inverting system, and becomes directly ready in a liquid form with collapsed polymeric chains included inside the water droplets of the W/O emulsion (Candau, 1990). Within a typical range from 15 to $25 \%$ of the final ingredient weight, the oil plays the complete role of "bio-based solvent" and final liquid vehicle as well as contributing to controlling polymerization exothermicity as continuous phase of the emulsion. When introduced 


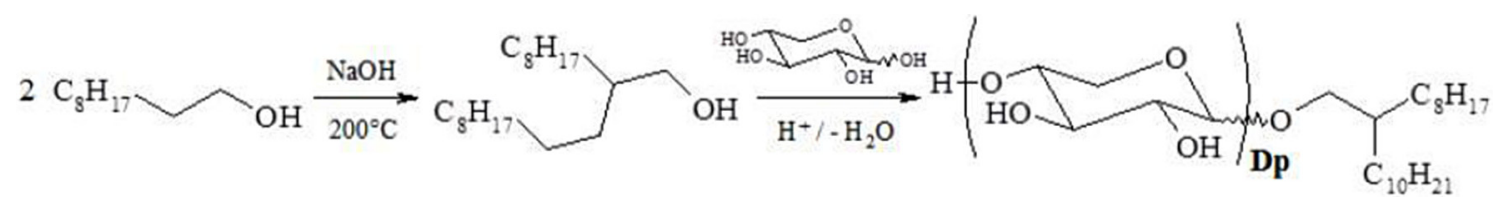

Fig. 8. Guerbet alcohol synthesis and glycosylation.

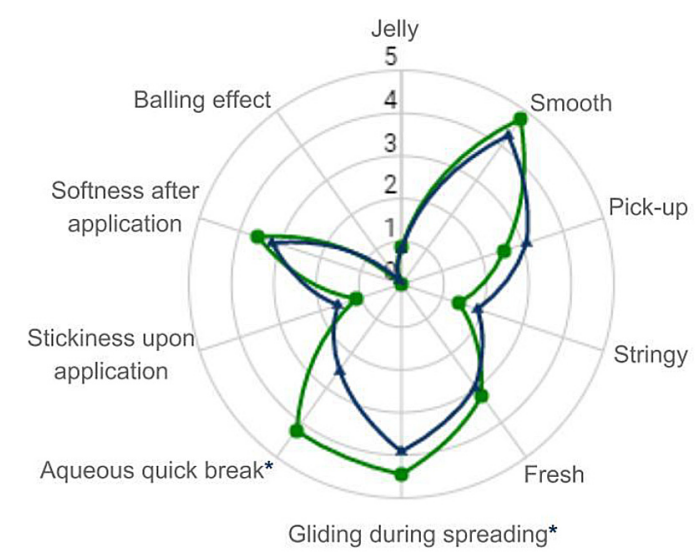

- Sodium Acrylate/Sodium Acryloyldimethyl Taurate Copolymer \& C15-19 Alkane $\Delta$ Sodium Acrylate/Sodium Acryloyldimethyl Taurate Copolymer \& Isohexadecane *Significant differences $(p<0.05)$

Fig. 9. Influence of oil on the sensory profile of liquid rheology modifiers.

into the water phase of a formula, due to the inverter agent included, the $\mathrm{W} / \mathrm{O}$ emulsion reverses and polymers expand spontaneously through electrostatic repulsions between the neutralized groups, thus giving a microgel network providing desired thickening effect. The swelling step is very quick and does not require high shear procedures. From a use level of $0.5 \%$ in water, the microgel network develops an elastic structure that will become condensed with increasing proportions, stabilizing added oils in different kinds of formulations (gel-based (Kuchekar and Bhise, 2012) or emulsions).

Of course, the oil nature influences the polymer skin feel perceived by the user (Merat et al., 2017). Very recently, one of the new biodegradable alkanes (mainly C15-16 in Tab. 1) combined with a plant-based inverting surfactant has allowed innovation towards a more sustainably designed liquid rheology modifier. This new ingredient (e.g. the Sepilife ${ }^{\text {TM }}$ range; INCI name: Sodium Acrylate/Sodium Acryloyldimethyl Taurate Copolymer \& C15-19 Alkane \& Polyglyceryl6 Laurate \& Polyglycerin-6) is characterized by a light skin feel with a high gliding effect. The skin feel is judged similar to nude skin by the expert sensory panelists, in line with the alkane original sensory profile. The influence of oil nature on the gliding effect and aqueous light skin feel is obviously visible (Fig. 9), in a sensory comparison performed by 10 expert panelists on the new liquid rheology modifier compared to previous ingredient with the same polymeric chain polymerized in mineral oil (Same gel-based formula at same viscosity). The new rheology modifier is $61 \%$ from natural origin according to ISO 16128 guidelines and is inherently biodegradable, which is a great improvement compared to previous liquid ones.

\subsection{Cosmetic formulations with a more sustainable manufacturing procedure}

Even if not fully bio-based, these two kinds of liquid ingredients contribute to reducing the environmental footprint of manufacturing processes as they give access to coldprocessable formulas, i.e. cream gels (1) and gel-in-oil formulations (2) e.g. patented Geltrap ${ }^{\mathrm{TM}}$ technology. Figure 10 shows a schematic description and the basic principle of the two formula concepts.

The newly developed liquid rheology modifier, obtained by inverse polymerization technology, used alone from a use level of $1 \%$ to $3 \%$, stabilizes $15 \%$ to $35 \%$ of added oil, depending on its nature, without the need for further additives. The formula containing polymer + oil + water, simply obtained by adding the polymer into the oil, then adding the water and mixing cold, is called cream-gel and meets the same stability criteria as a traditional $\mathrm{O} / \mathrm{W}$ emulsion. Stability is supported by the polymer-dense microgel network and corresponding suitable rheology profile.

Gel-in-Oil is a type of cold-processable High Internal Phase W/O emulsion (HIPE), obtained by a synergistic effect between the two previously described ingredients: a liquid rheology modifier and a liquid W/O emulsifier (Cambos et al., 2014). Polymer rheology profile is also key for gel-in-oil stability. The formula typically contains 2 to $4 \%$ of liquid emulsifier, 6 to $11 \%$ of added oil, 1 to $1.3 \%$ of liquid rheology modifier, and 1 to $3 \%$ of a polyol that plays the role of antifreeze agent like in classical $\mathrm{W} / \mathrm{O}$ emulsions and water. Gel-in-oil emulsions are made simply by first adding polymer to water to create the gel, then adding the fatty phase including oils and the liquid-optimized emulsifying base, then mixing (Mixing from paddle mixers to high shear is possible.); complete inversion of the gel is obtained after few minutes. Long-term stability is provided by the quality of the interfacial film coming from this surfactant blend and reinforced by the reduced mobility of gelled droplets. Looking to benefits for consumers, sensoriality and strong emollience effects are the major distinctive assets of gel-in-oil. On one hand, the unique sensory profile combines a first freshness with a second cushion effect and a comfortable final sensation. On the other hand, gel-in-oil technology provides similar emollience sensations as classical $\mathrm{O} / \mathrm{W}$ and $\mathrm{W} / \mathrm{O}$ emulsions with a much lower oil amount.

The impact of the manufacturing process on energy consumption and production time has been calculated on a pilot procedure basis $(5 \mathrm{~kg}$ of formula - Trimix $10 \mathrm{~L}$ 


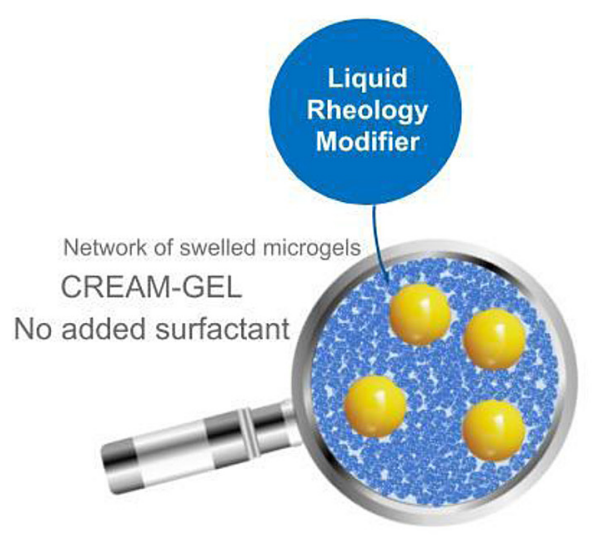

Oil phase up to $35 \%$

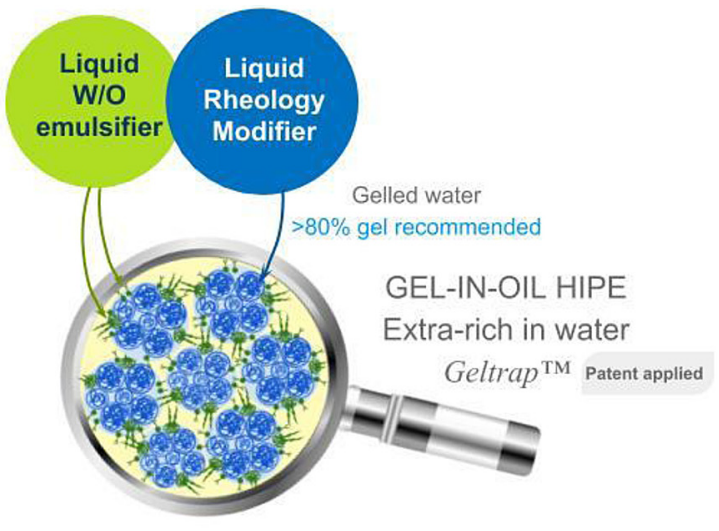

Oil phase $8-15 \%>$ Emolience with low oil \%

Fig. 10. Responsible formula technologies: cream-gel and gel-in-oil - basic principle.

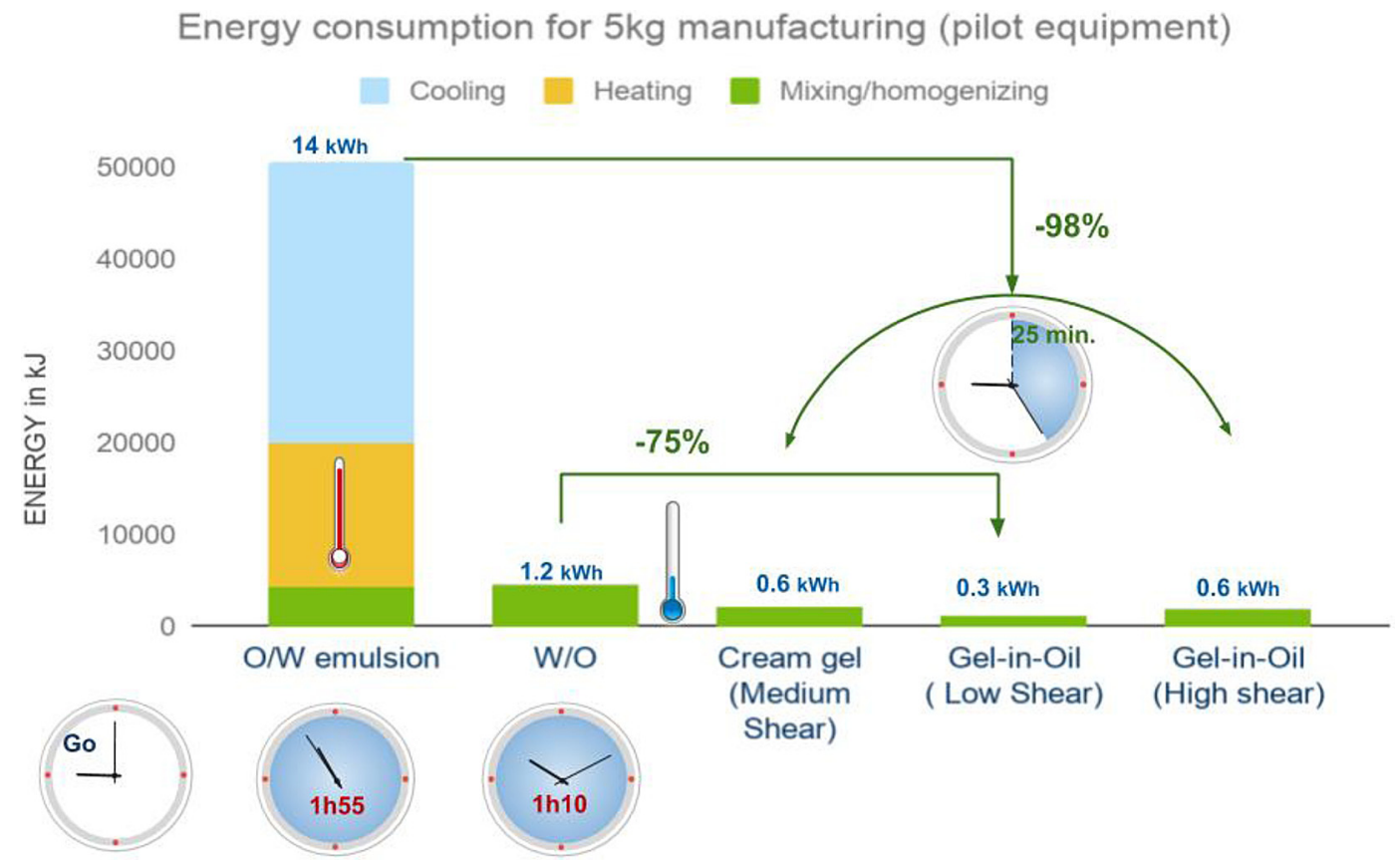

Fig. 11. Impact of manufacturing process on energy consumption and production time.

equipment from VMI Rayneri). The full energetic power of each mixing device was used as the default value. In this context, the compared results, shown in Figure 11, demonstrate the benefit of the cream-gel and gel-in-oil concepts. Timesaving with gel-in-oil and cream-gel is highly significant compared to classical emulsions, moving from almost two hours for hot process $\mathrm{O} / \mathrm{W}$ emulsion to 25 minutes, even when compared to $\mathrm{W} / \mathrm{O}$ emulsion manufactured cold. There is in fact no need of progressive addition of the oily phase with the gelin-oil or the cream gel; addition can be in one shot. Globally, the energy consumption during pilot manufacturing of the gelin-oil and cream gel is reduced by around 97\% compared with the $\mathrm{O} / \mathrm{W}$ emulsion with a hot process and by around $70 \%$ compared with the cold $\mathrm{W} / \mathrm{O}$ emulsion process.

These two concrete examples emphasize the importance of lipid structure in the design of ready-and easy-to-use specialty ingredients and the further development of responsible formula technologies for cosmetic producers. Cream gels and gel-in-oil are available alternatives to traditional $\mathrm{O} / \mathrm{W}$ and $\mathrm{W} / \mathrm{O}$ emulsions.

\section{Lipids, pillars for bio-based surfactant development}

Bio-based lipids are also key components in the design and development of environmentally friendly surfactants because these molecules are amphiphilic, having a polar head and a lipophilic tail. Consequently, hydrocarbon chains are an integral part of the surfactants' chemical structures. The natures of the polar head, chain length, unsaturations and chemical functions will define the Hydrophilic-Lipophilic Balance (HLB) and also the final applications of surfactants. As examples, for short chains $(<\mathrm{C}-8)$, expected final physical 


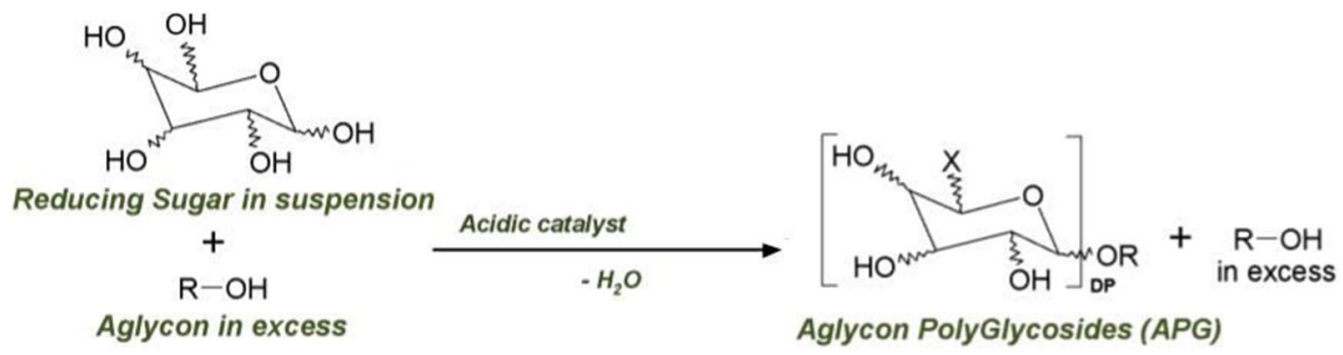

Fig. 12. Fischer's glycosylation of fatty alcohol by a sugar.

and chemical properties will be solubilizing and detergent properties. For intermediate chains comprised between C-8 and $\mathrm{C}-12$, it will be foaming properties. And for long chains ( $>\mathrm{C}-12$ ), it will be emulsifying properties.

From fatty acids or methyl esters, the most popular biosurfactants are sorbitan esters, saccharose esters, (oligo)glycerol esters and betaines. Their industrial processes involve esterification reactions with acidic catalysts or basic activation, with or without organic solvents. The linkers between the polar heads and the chains are ester bonds. Polyesterification reactions (or degree of esterification) have to be controlled to target the suitable HLB and the resulting desired performance.

From fatty alcohols, AlkylPolyGlycosides are probably the best example of $100 \%$ bio-based surfactants available on the market. They are produced by performing glycosylation reactions according to the Fischer's conditions and using a reducing sugar. Today, the most widely used sugar is glucose coming from wheat or corn starch leading to Alkyl PolyGlucosides (APG). The second reducing sugar used is xylose from wood hemicelluloses leading to Alkyl PolyXylosides (APX). On the industrial scale, the one-step process is quite simple and consists in dispersing the reducing sugar in a molar excess of fatty alcohols (reactant and solvent) with an acidic catalyst (Fig. 12). The removal of water by heating and vacuum allows APG formation. Secondary reactions are very limited, and reducing sugar oligomerization occurs during the glycosylations. The mean polymerization degrees (PD) of the APG are between 1.0 and 1.5. At the end of glycosylations, the catalyst is neutralized and the fatty alcohol excess is either removed by wipe film evaporation (solubilizing or foaming surfactants in aqueous solutions) or remains in the final surfactant. This second work-up is preferably dedicated to emulsifying surfactants, as fatty alcohols also play a role in the emulsions' stabilization and sensoriality.

In the domain of emulsifiers, the impact of fatty chain length on the final emulsion type $(\mathrm{O} / \mathrm{W}, \mathrm{W} / \mathrm{O})$ and emulsion stability has been extensively explored.

\section{Lipid selection in oil-in-water glucolipid emulsifiers for distinctive performance and sensoriality}

Due to their amphiphilic structure, emulsifiers can set up varied organization in solution, such as liquid crystals, intermediate organizations between a liquid and a solid, which are interesting for cosmetic applications. Alkylpolyglucoside emulsifiers, paired with fatty alcohols with the same chain length, are known for their versatile ability to create lamellar liquid crystals (Savic et al., 2004) involved in $\mathrm{O} / \mathrm{W}$ emulsion stabilization mechanisms. APG develop two types of structures due to the synergistic effect between surfactant and free fatty alcohol: firstly lamellar bilayers around the oil droplets, also called onion-type organization (Fig. 13 visualized by birefringent Maltese cross structures), which form kinds of "shells" protecting oil droplets from coalescence; secondly a lamellar tridimensional network in the continuous aqueous phase acting as an elastic structure (typical elastic character measured by rheology oscillatory experiments). In addition, with classical surfactant interface effect, lamellar structures help to stabilize oil droplets (Tasic-Kostov et al., 2012), and explain the powerful emulsifying properties of APG, especially with plant oils, well-known to be difficult to stabilize. Despite the fact that liquid crystal development is common to APG, it has been observed as a more robust organization for longer fatty chains, from $\mathrm{C} 16$ to $\mathrm{C} 22$ with a stronger stabilizing effect, especially when the emulsion is stored between $40-50{ }^{\circ} \mathrm{C}$. Longer chains provide consistently higher elastic character to the emulsions. Emulsifiers with $\mathrm{C} 20$ to $\mathrm{C} 22$ chains are especially efficient in stabilizing oil from lower concentrations, such as 0.5 to $1 \%$, whereas $2 \%$ is required for shorter lipophilic chains.

Apart from emulsion stability, lamellar liquid crystals further impart two main specific benefits from the consumer perspective:

- firstly a lamellar structure is similar to lipid organization in the stratum corneum: the emulsions are naturally biocompatible (Bouwstra et al., 2002; Rodríguez-Abreu and Lazzari, 2008; Tasic-Kostov et al., 2010);

- secondly, due to free OH groups in APG and free alcohols, hydrogen bonds can form with water molecules which become "entrapped" in the lamellar phases. Therefore, lamellar phases act as water reservoir and impart intrinsic moisturizing potential (LeHen-Ferrenbach and Hill, 2008; Savic et al., 2011). As an example, the moisturizing effect can be highlighted in vivo by corneometry measurement through a significant increase of skin capacitance compared to the untreated area. Very simple $\mathrm{O} / \mathrm{W}$ emulsions, containing the APG emulsifier $(3 \%)$, a plantbased ester: cetearyl ethyllhexanoate $(20 \%)$, a liquid rheology modifier $(0.5 \%)$ and water, provide significant moisturizing benefits, even 5 hours after application on the skin (Fig. 14), versus the comparative formula without APG. As can be noticed by the candlestick appearance of Figure 13, some variations may occur in the moisturizing 


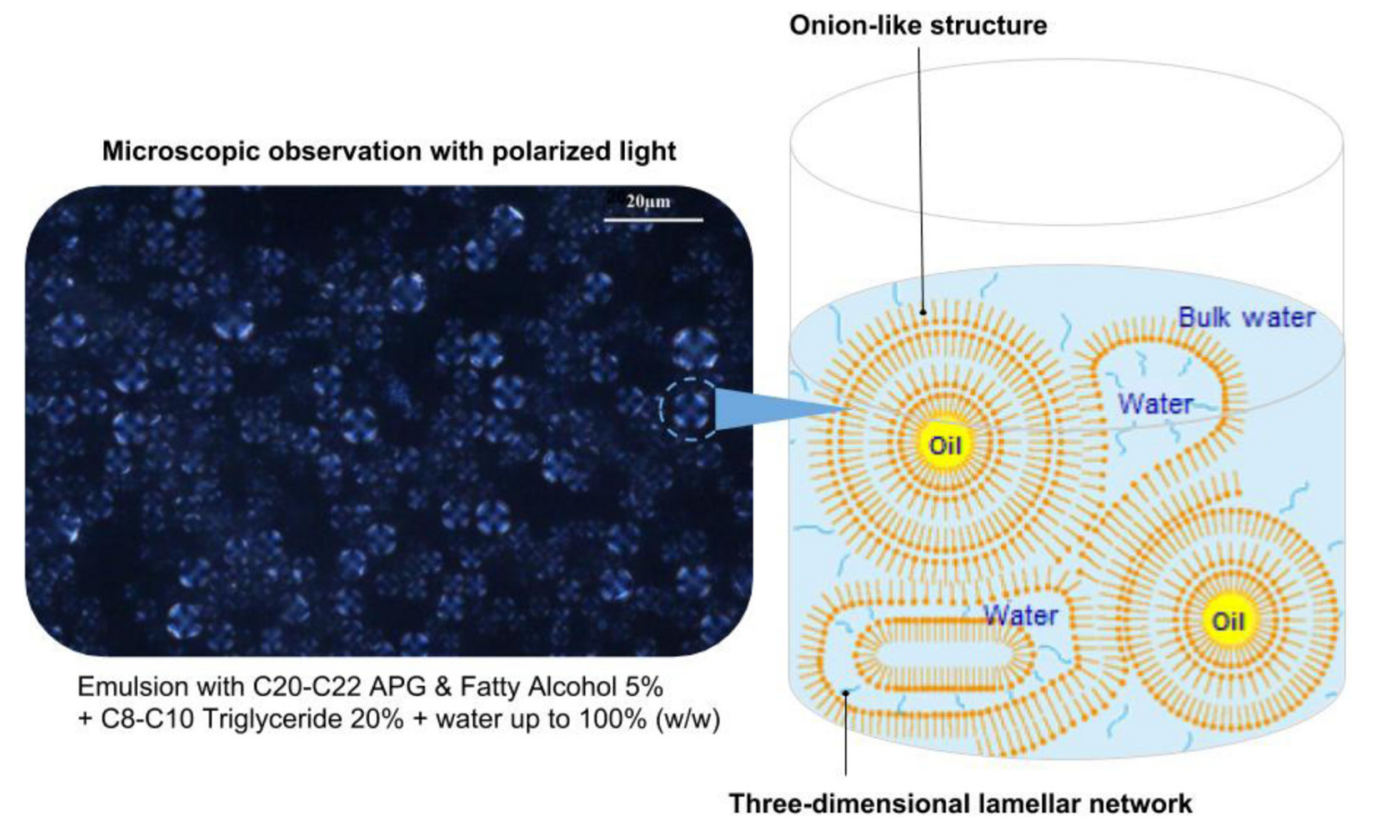

Fig. 13. Lamellar phase schematic organization in O/W APG's emulsion.

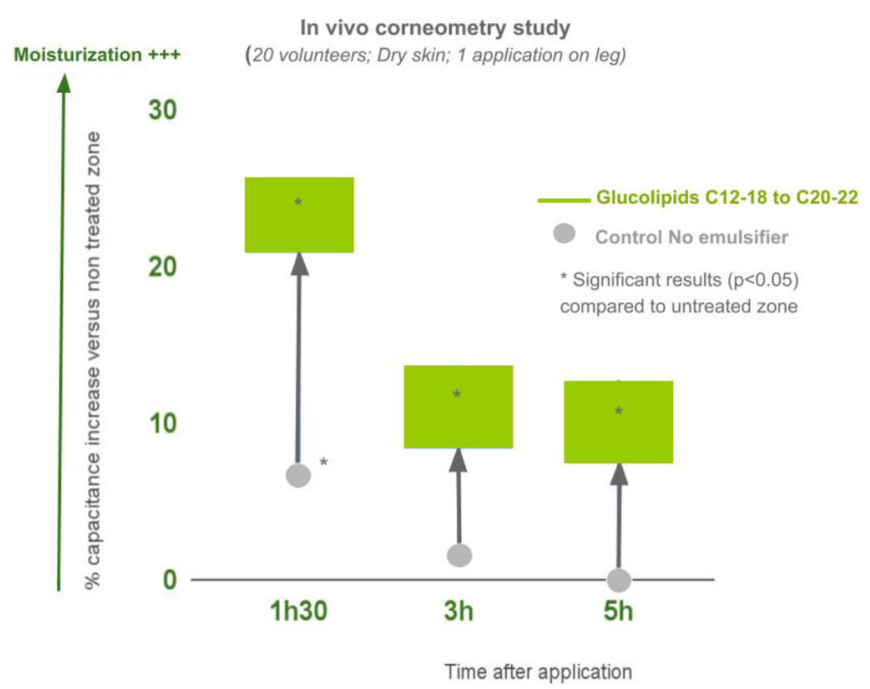

Fig. 14. Influence of lipids on in vivo moisturizing effect.

effect according to the chain length chosen for the APG emulsifier and the resulting ratio between onion-like structures and a lamellar phase network in the continuous aqueous phase.

As another benefit for consumers, APG emulsifiers offer also a wide range of emulsion textures, from white to very white opaque emulsions, from very fluid to consistent creams coming with various sensory profiles, from light to rich skin feel. As illustrated in Figure 15, the selection of the lipid part has a great impact, both on the emulsion consistency and on the skin feel in comparable conditions. Broad cuts of fatty alcohol, including short C12 chains, produce versatile textures from very fluid and sprayable emulsions to creams depending on proportions and other additives. Specific attention should be paid to the ratio between short and long chains to get a good compromise between emulsion fluidity (expected from the short chains) and stability (provided by the long chains). For instance, myristyl glucoside and myristyl alcohol act mainly as a co-emulsifier and a consistency agent, since their stabilizing power is not sufficient to be used a sole emulsifier. On the contrary, narrow cuts with long chain fatty alcohols mainly give consistent textures, especially with C16-C18 sections.

Determining a clearly understood relationship between emulsifier structure and sensory profile is more difficult as sensory perception is a multiparametric phenomenon. Some sensory characteristics are shared, such as excellent spreadability, rather linked to constant shear thinning profile of the emulsions, and soft skin finish, whatever the lipid structure. However, reproducible observations have been made on a multitude of different emulsions showing some obvious influence of fatty chain length: APG designed with high C16 to C18 fatty alcohol contents provide a rich skin feel whereas APG designed with high C20 to C22 fatty alcohol contents provide a light skin feel. The APG lipid structure is key in fitting to skin target and customer satisfaction.

Owing to their robust chemical structure including glycosidic linkage, APG emulsifiers benefit from a perfect stability in extreme $\mathrm{pH}$ conditions, as well as perfect compatibility with oxidant molecules such as hydrogen peroxide. This perfect stability against hydrolysis opens perspectives in bleaching or hair color formulations. Moreover, for long fatty chains, the rheology profile, combining shear thinning and elastic character, can support a good distribution from root to tip and contributes to sheathing hair fiber effect by preventing product run-out. APG emulsifiers are thus interesting candidates for leave on hair treatments such as masks or products with pause time as well as mascaras. 


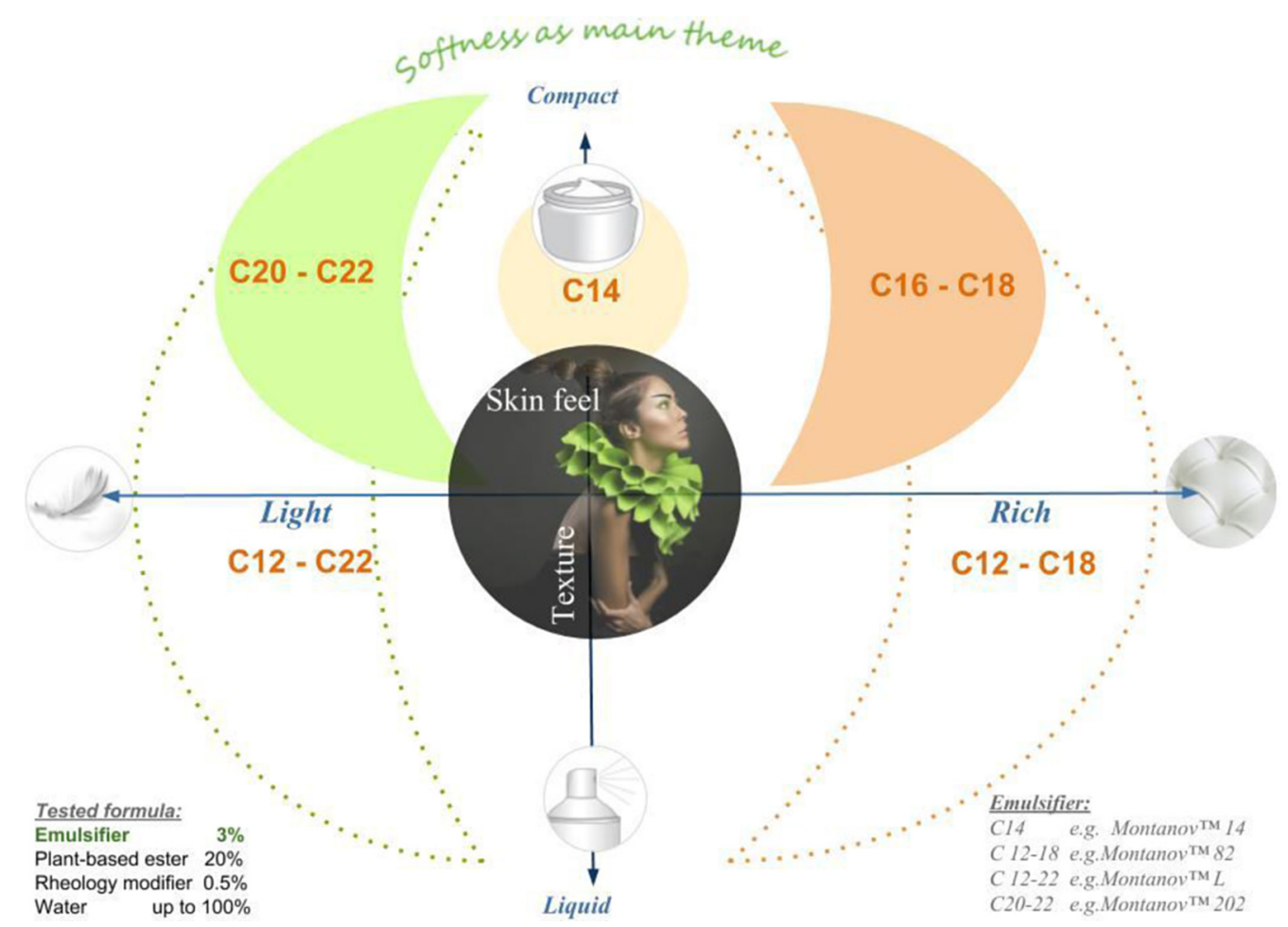

Fig. 15. Influence of fatty chain length on final emulsion sensoriality.<smiles></smiles>

Amino acid<smiles>[R1]C(=O)Cl</smiles>

Acyl chloride

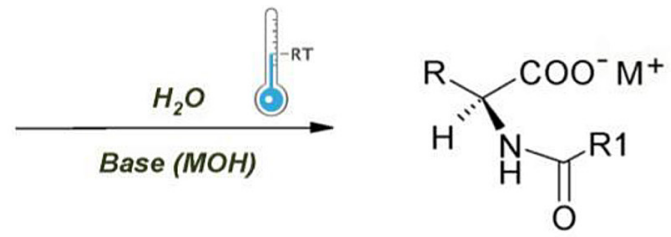

LipoAminoAcid (LAA)

Fig. 16. Schotten-Baumman acylation.

\section{Lipid selection, driver of active ingredients efficacy}

Lipoaminoacids (LAA) are anionic biosurfactants with an amino-acid derived polar head. They are obtained by carrying out Schotten-Baumman's acylation reactions on pure or mixed amino acids and acyl chlorides. Despite the chlorine atom, acyl chlorides are preferred to carboxylic acids or methyl esters because of their higher reactivity at room temperature. Schotten-Baumman acylations consist in adding acyl chloride to an aqueous solution of amino acids with the $\mathrm{pH}$ around 10 (Fig. 16). This process creates an amide bond linking the amino acid and the fatty chain. The reactions are quite simple and the main difficulty is in controlling the hydrolysis of the chloride acid to minimize the quantity of fatty acids in the final products. From an environmental point of view, the advantages are the use of water as solvent, low energy consumption (the reactions occur at room temperature), the absence of sophisticated catalysts and good atom economy. The main drawback is the use of acyl chlorides, which come from oleochemistry but need for their preparation the use of nonenvironmentally friendly chlorinated agents such as thionyl chloride or $\mathrm{PCl}_{3}$.

At the end of the acylations, the reaction media are composed of LAA, fatty acids and salts. Two work-ups are then possible: the first one with only a neutralization at $\mathrm{pH}$ around 7 and adjustment of dry matter. In this case, the final products are aqueous solutions of neutral LAA with foaming properties; the second work-up causes precipitation by adjusting the $\mathrm{pH}$ below 2 and removal by filtration and washing of all the salts generated during the acylation. With this second work up, the final LAA are isolated as powders after drying with a higher purity. For this reason, they are preferably dedicated to cosmetic actives. These amphiphilic structures are interesting biological vectors of amino acids.

Taking into account the number of amino acids encoded by DNA and the number of acyl chlorides available in the market, the synthesis of a large range of LAA is possible. Unfortunately, it is very difficult to predict relationships between LAA chemical structure and their physical, chemical or biological properties. As a consequence, a screening 


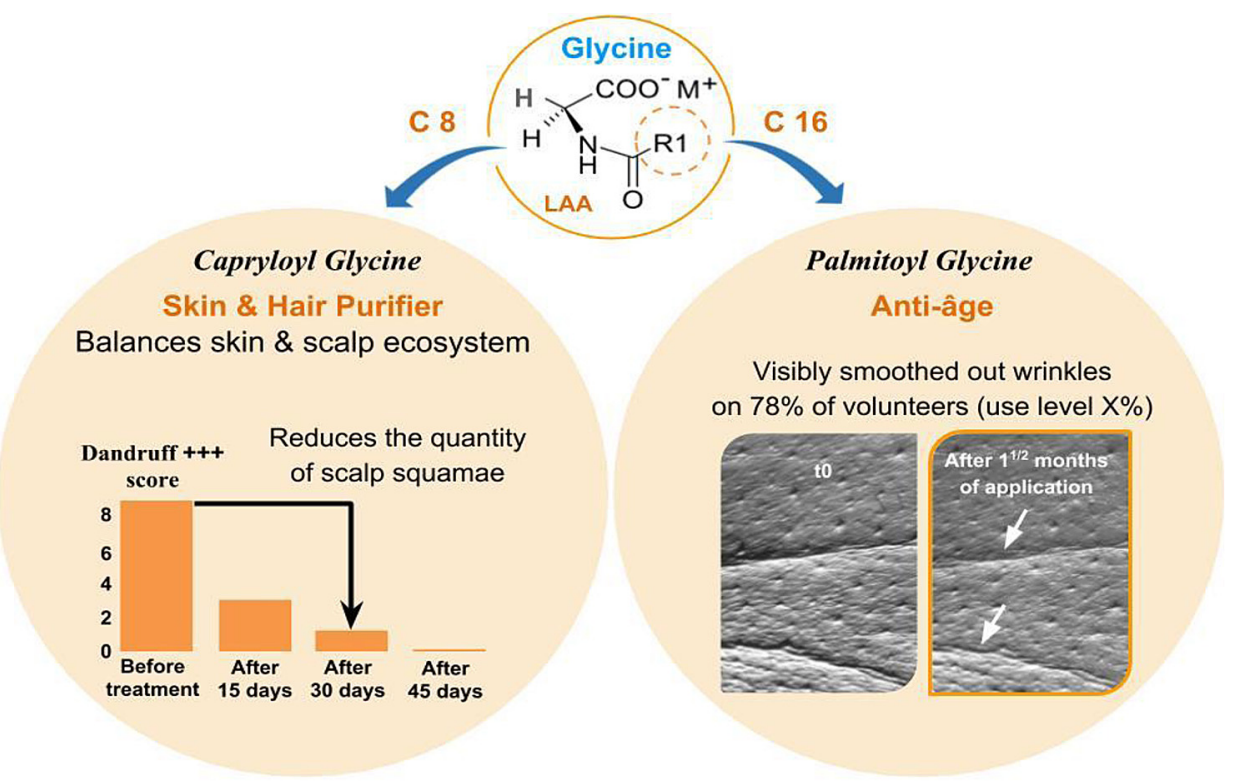

Fig. 17. Influence of fatty chain length on in vivo lipoaminoacid biological efficacy.

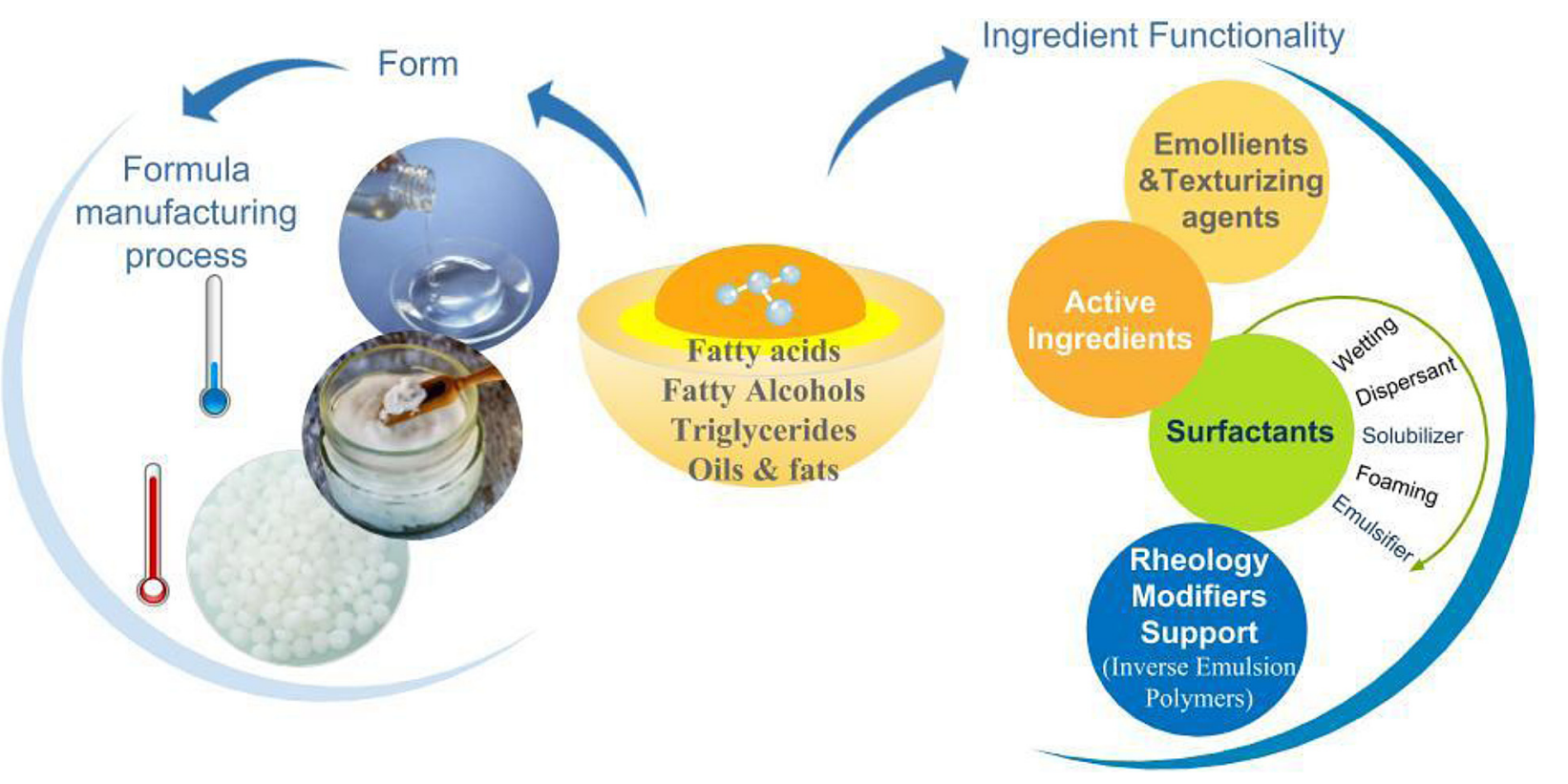

Fig. 18. Lipids' pivotal role.

methodology is useful to identify and develop new purified LAA with efficient biological activities. Nevertheless, we do observe that the lipid portion plays an essential role in the final biological efficacy.

As an illustrating example, two lipoaminoacids made from glycine but with two different lipid chains are compared: capryloyl glycine (Lipacide $^{\mathrm{TM}} \mathrm{C} 8 \mathrm{G}$ ) and palmitoyl glycine (Timecode ${ }^{\mathrm{TM}}$ ). In vitro and in vivo biological evaluations show very different activity (Fig. 17).

Capryloyl glycine limits the proliferation of germs responsible for acne-prone skin and dandruff such as Staphylococcus aureus, Staphylococcus epidermidis, Propionibacterium acnes and Pityrosporum ovale. Moreover this molecule acts as a skin buffer and helps to maintain or to restore the natural acidic $\mathrm{pH}$ of healthy skin. Capryloyl glycine can thus be classified as a skin and scalp purifier. For instance, visible scalp benefits are confirmed in vivo when tested at $2 \%$ in a shampoo, used once every 3 days by 40 volunteers with capillary seborrheic dermatitis. Positive effects starting after 15 days of use and complete return to a healthy scalp state are obtained after 1 month. Sebaceous secretion is reduced, there is no more dandruff, and concurrent scalp irritation has disappeared.

For the other example, in vitro investigations demonstrate that palmitoyl glycine acts on skin cell metabolism and rejuvenates aging tissues, both by boosting dermal organiza- 
tion (with among others collagen network weakening with age) and reducing an aging-associated hyper-inflammatory state, also referred to as "inflamm-aging" (Perez et al., 2009). A final wrinkle corrector effect is confirmed in vivo when tested at $2 \%$ in a cream, applied 2 times a day on crow's feet, by 20 volunteers with an average age of 56 . The number of deep and medium wrinkles as well as the total wrinkle area is significantly reduced after 1.5 months of application, more efficiently than with the same formulation containing $3 \%$ of an anti-aging peptide reference.

\section{Conclusion}

Lipids are essential in controlling the properties of speciality ingredients and innovating in broad categories such as biodegradable emollients, environmental friendly surfactants, active ingredients, and more sustainable, easy-to-use rheology modifiers.

Sustainable sourcing from various plant origins, through more or less complex transformations, leads to great lipid molecular diversity. These can be used directly as cosmetic ingredients or as raw materials to create advanced cosmetic ingredients. By controlling both ingredient functionality and final ingredient form (liquid to solid), lipids play a central role (summary in Fig. 18), enabling the further development of cosmetic formulation concepts, saving time and energy at the production level.

Considering this expertise, we foresee a great future for oleochemistry. Creativity in transformation processes will surely remove existing technical barriers and lead to fully environmentally friendly ingredient manufacturing processes. Looking at original lipid sourcing, such as microalgal lipids (Bellou et al., 2014), and biotechnologies available at industrial scales will also open paths to new ingredients with augmented performance and renewed sensoriality.

\section{References}

Bellou S, Baeshen MN, Ellazazzy AM, Aggeli D, Sayegh F, Aggelis G. 2014. Microalgal lipids biochemistry and biotechnological perspectives. Biotechnol. Adv. 32(8): 1476-1493.

Bouwstra JA, Gooris G, Ponec M. 2002. The lipid organisation of the skin barrier: liquid and crystalline domains coexist in lamellar phases. J. Biol. Phys. 28: 211-223.
Cambos S, Clemenceau F, Amalric C. 2014. Revival of water-in-oil formulation concept with better producer and consumer benefits. Sci. Beauty 4(3): 58-63.

Candau F. Polymerization in inverse emulsion and microemulsion. In: Candau F, Ottewill RH, eds. An introduction to polymer colloids. Dordrecht: Kluwer Academic, 1990, pp. 73-96.

Kuchekar S, Bhise K. 2012. Formulation and development of antipsoriatic herbal gelcream. J. Sci. Industrial Res. 71(4): 279284.

Latshaw AK. Analysis of cocoa content in chocolate using triborheometry and its correlation to mouthfeel. The Society of Rheology, 86th annual meeting, 2014, October, Philadelphia.

LeHen-Ferrenbach C, Hill K. 2008. Sugar-based surfactants for consumer products and technical applications. In: Sugar-based surfactants. Boca Raton, Florida: CRC Press.

Merat E, Roso A, Sourdon C, Gomez Y, Santos F. 2017. Psychorheology, insight on polymers. Sci. Beauty 6(4): 42-46.

Nonomura Y, Arashi Y, Maeno. 2009. How do we recognize water and oil through our tactile sense? Colloid. Surf. B Biointerfaces 73: $80-83$.

Perez C, Dumont S, Cattuzzato L, Trouve G, Chevrot N, Stoltz C. 2009. A novel high performance wrinkle corrector with anti inflamm-aging properties. J. Cosmet. Sci. 60(5): 552-553.

Rodríguez-Abreu C, Lazzari M. 2008. Emulsions with structured continuous phase. Colloid Interface Sci. 13(4): 198-205.

Savary G, Grisel M, Picard C. 2013. Impact of emollients on the spreading properties of cosmetic products: A combined sensory and instrumental characterization. Colloid. Surf. B Biointerfaces 102: 371-378.

Savic S, Vuleta G, Daniels R, Müller-Goymann C. 2004. Colloidal microstructure of binary systems and model creams stabilized with an alkylpolyglucoside non-ionic emulsifier. Colloid Polym. Sci. 283(4): 439-451.

Savic S, Lukic M, Jaksic I, Reichl S, Tamburic S, Müller-Goymann C. 2011. An alkyl polyglucoside-mixed emulsifier as stabilizer of emulsion systems: The influence of colloidal structure on emulsions skin hydration potential. J. Colloid. Interface Sci. 358: 182-191.

Tasic-Kostov M, Savic S, Lukic M, Tamburic S, Pavlovic M, Vuleta G. 2010. Lactobionic acid in a natural alkylpolyglucoside-based vehicle: assessing safety and efficacy aspects in comparison to glycolic acid. J. Cosmet. Dermatol. 9: 3-10.

Tasic-Kostov M, Pavlovic D, Lukic M, Jaksic I, Arsic I, Savic S. 2012. Lactobionic acid as antioxidant and moisturizing active in alkyl polyglucoside-based topical emulsions: the colloidal structure, stability and efficacy evaluation. Int. J. Cosmet. Sci. 34: 424-434.

Cite this article as: Duprat-de-Paule S, Guilbot J, Roso A, Cambos S, Pierre A. 2018. Augmented bio-based lipids for cosmetics. OCL 25(5): D503. 